\title{
Role of microRNA 146a on the healing of cornea alkali burn treated with mesenchymal stem cells
}

\author{
$\mathrm{XU} \mathrm{LUO}^{1^{*}}$, JIANMIN LI $^{2^{*}}$, LIHUI YIN $^{3}$, JIAN PAN $^{4}$, YANG ZHANG $^{4}$ and ZIPEI JIANG ${ }^{4}$ \\ Departments of ${ }^{1}$ Wounds and Burns, and ${ }^{2}$ Pathology; ${ }^{3}$ Laboratory of Internal Medicine; \\ ${ }^{4}$ Department of Ophthalmology, The First Affiliated Hospital of Wenzhou Medical University, \\ Wenzhou, Zhejiang 325000, P.R. China
}

Received July 4, 2016; Accepted June 9, 2017

DOI: $10.3892 / \mathrm{mmr} .2018 .9328$

\begin{abstract}
The aim of the present study was to investigate the effect of microRNA 146a (miR146a) on promoting the repair of corneal alkali burn with bone marrow mesenchymal stem cells (MSCs). A total of 24 Sprague-Dawley female rats were divided into a normal group (Control), a normal MSC treatment group (Normal MSCs), an miR146a knockout MSC treatment group (miR146a-low MSCs) and an miR146a high-expression MSC treatment group (miR146a-high MSCs) according to the random number table. Quantitative polymerase chain reaction was used to evaluate the expression levels of miR146a. MTT assay was performed to measure the cell viability of mesenchymal stem cells (MSCs) and apoptosis was measured by flow cytometry. The expression levels of p65 nuclear factor $(\mathrm{NF})-\kappa \mathrm{B}$, proliferating cell nuclear antigen (PCNA) and Fas proteins were analyzed by western blotting. MSCs were tested for the secretion levels of vascular endothelial growth factor (VEGF), CD45, interferon (IFN)- $\gamma$ and interleukin (IL)-10 by ELISA. The miR146a-high MSCs improved cell viability of MSCs and inhibited apoptosis of MSCs following alkali burn. miR146a-high MSCs decreased the expression levels of p65NF- $\kappa \mathrm{B}$ and PCNA, and enhanced the expression level of Fas. Furthermore, miR146a-high MSCs improved the cornea opacity and enhanced the inhibition of neovascularization in the rats following alkali burn. miR146a-high MSCs inhibit the expression of VEGF, CD45, IFN- $\gamma$, while enhanced the expression of IL-10. Therefore, miR146a promotes the repair of corneal alkali burn in rats treated with MSCs.
\end{abstract}

Correspondence to: Dr Zipei Jiang, Department of Ophthalmology, The First Affiliated Hospital of Wenzhou Medical University, Nanbaixiang, Ouhai, Wenzhou, Zhejiang 325000, P.R. China E-mail: jzp20012000@163.com

${ }^{*}$ Contributed equally

Key words: microRNA 146a, mesenchymal stem cells, p65 nuclear factor- $\kappa \mathrm{B}$, alkali burn

\section{Introduction}

Ocular alkali burn is a common intractable ocular disease in the clinic, which may cause blindness (1). Infiltrating leukocytes following alkali burn release proteolytic enzymes and a variety of inflammatory mediators, which leads to non-specific damage on the corneal tissue, seriously affecting the structure and function of the cornea. Corneal alkali burns cause serious conditions, such as corneal melting, neovascularization and ulcer perforation (2). Severe alkali burns specifically affect the vision of these patients (3). Chemical burns, particularly alkali burns, are a common cause of corneal neovascularization (CNV), and CNV is closely associated with vision loss. Numerous studies have investigated the therapeutic methods that may cure corneal alkali burns quickly and effectively $(4,5)$.

Mesenchymal stem cells (MSCs) are derived from adult stem cells in the mesoderm, which is an important cellular component of the hematopoietic microenvironment $(6,7)$. MSCs contribute to proliferation and differentiation of a variety of tissues, such as bone, cartilage, muscle, ligaments, tendons and adipose stromal cells, and have low immunogenicity. Therefore, MSCs are considered to be an ideal cellular source for tissue engineering $(8,9)$. In addition, MSCs are easily transfected and carry exogenous genes. Furthermore, MSCs have a wide range of applications in cell and gene therapy (10). Currently, clinical trials indicate that MSCs may be used to repair genetic deficiency diseases of mesenchymal tissue, which presents broad clinical application possibilities $(11,12)$.

Micro RNA (miRNA) is a class of non-coding, single-stranded miRNA (18-24 bp), which incorporates into the RNA-induced silencing complex, adjusting the stability and translational efficiency of target molecules, and effectively inhibiting gene expression $(13,14)$. In recent years, studies have demonstrated that miRNA is particularly important in post-transcriptional regulation of embryonic development, phylogeny, tissue differentiation and evolution of disease, for example, cancer, cardiovascular disease and neurological diseases (15). In the process of MSC osteogenic differentiation, miRNAs are also vital.

In the current study, MSCs were genetically modified using a replication lentivirus over- or under-expressing miR146a genes. We hypothesize that the MSCs over-expressing miR146a are able to effectively repair the corneal alkali burn. 
The present study may provide a promising method for corneal alkali burn treatment.

\section{Materials and methods}

Isolation and culture of MSCs. A total of one six-week-old, female Sprague-Dawley (SD; $100 \mathrm{~g}$ ) rat was purchased from Animal Experimental Center of Wenzhou Medical University (Wenzhou, China) and sacrificed immediately to obtain the bone marrow. Bone marrow cells were obtained by flushing the femurs and tibias with Dulbecco's modified Eagle's medium (DMEM; Gibco; Thermo Fisher Scientific, Inc., Waltham, MA, USA; cat. no. 11965118). The cells were cultured in culture flasks in complete DMEM with $10 \%$ fetal bovine serum (FBS; Gibco; Thermo Fisher Scientific, Inc.; cat. no. 16000044$)$ and penicillin/gentamycin $(10 \mathrm{mg} / \mathrm{ml}$; Gibco; Thermo Fisher Scientific, Inc.; cat. no. 15070063$)$ at $37^{\circ} \mathrm{C}$. After $72 \mathrm{~h}$, the nonadherent cells were removed by replacing the DMEM. The DMEM was refreshed every three days, and the cells were transferred upon reaching $80 \%$ confluence. MSCs were isolated using flow cytometry, and MSCs from the third passage were collected and resuspended in phosphate-buffered saline (PBS) with 10\% FBS. Monoclonal antibodies were added for $30 \mathrm{~min}$ at $4^{\circ} \mathrm{C}$, including cluster of differentiation (CD)90 (Abcam, Cambridge, UK; 1:1,000; cat. no. EPR3132); CD45 (Abcam; 1:600; cat. no. ab10558); CD34 (Abcam; 1:3,000; cat. no. ab81289); CD73 (Abcam; 1:600; cat. no. ab175396). The phycoerythrin-conjugated antibodies against cluster of differentiation CD90, CD45, CD34 and CD73 were purchased from Biolegend, Inc. (San Diego, CA, USA). PBS served as a negative control.

Animal model. A total of 24 female SD rats were used in the current study, rats (age, 8-12 weeks; weight, 200-220 g) were purchased and housed in an environmentally-controlled breeding room of Wenzhou Medical University (Wenzhou, China), at a temperature of $20 \pm 2^{\circ} \mathrm{C}$, a relative humidity of $60 \pm 5 \%$ and under a 12 -h light/dark cycle. The rats were anesthetized by intraperitoneal injection of chloral hydrate $(10 \%$; $4 \mathrm{ml} / \mathrm{kg})$. Sodium hydroxide $(\mathrm{NaOH} ; 4 \mu \mathrm{l}, 1 \mathrm{~mol} / \mathrm{l})$ was applied to a piece of filter paper (diameter, $3 \mathrm{~mm}$ ) and placed in the center of the cornea for $40 \mathrm{sec}$. The cornea was immediately rinsed with saline for $1 \mathrm{~min}$. The animals were administered a subconjunctival one-off injection of MSCs $\left(1 \times 10^{7}\right.$ cells) through the tail vein. The present study was approved by the veterinary ethics committee of Zhejiang (Wenzhou, China; ID: LY16H120004).

Quantitative polymerase chain reaction $(q P C R)$. $\mathrm{qPCR}$ was performed as previously described (16). mRNA was obtained from the MSCs using an RNeasy kit (Qiagen China Co., Ltd., Shanghai, China) and cDNA was synthesized from total RNA (TaqMan ${ }^{\mathrm{TM}}$ Fast Reagent Starter kit, cat. no. 4352407; Thermo Fisher Scientific, Inc.). PCR was performed with the following thermocycling conditions: An initial $5 \mathrm{~min}$ at $95^{\circ} \mathrm{C}$, followed by 40 cycles of $95^{\circ} \mathrm{C}$ for $30 \mathrm{sec}, 55^{\circ} \mathrm{C}$ for $30 \mathrm{sec}$ and $72^{\circ} \mathrm{C}$ for $30 \mathrm{sec}$. The primer sequences were as follows: Forward, $5^{\prime}-\mathrm{ACC}$ ACACCTTCTACAATGA-3' and reverse, 5'-ATAGCACAG CCTGGATAG-3' for $\beta$-actin, which were designed with primer premier 6.0 (Premier Biosoft International, Palo Alto, CA, USA). qPCR was conducted with an Applied Biosystems 7500 real-time PCR system. Results were analyzed using the Light Cycler Software version 3.5. Housekeeping gene $\beta$-actin was used as an internal reference to normalize the results. All experiments were performed in triplicate. Finally, the $2^{-\Delta \Delta \mathrm{Cq}}$ method was performed to calculate the relative expression (17).

MTT assay. Evaluation of cell viability of MSCs was performed via MTT assay. MSCs $\left(2 \times 10^{5}\right.$ in $\left.100 \mu \mathrm{l}\right)$ were seeded into 96-well cell plates and cultured in a cell incubator at $37^{\circ} \mathrm{C}$ overnight. Then, $10 \mu 1$ MTT $(5 \mathrm{mg} / \mathrm{ml})$ was added to each well and the plates were incubated for $3 \mathrm{~h}$ at room temperature in the dark. The dimethyl sulfoxide (DMSO) was added and the medium was discarded. The absorbance $490(\mathrm{~nm})$ was tested 3 min later using an ELISA microplate reader.

Apoptosis analysis of MSCs. Apoptosis analysis was performed as described previously (18). Briefly, MSCs were harvested from the cell culture flasks at approximately $80-90 \%$ confluence and centrifuged at $300 \mathrm{xg}$ for $10 \mathrm{~min}$ at $4^{\circ} \mathrm{C}$. The medium was discarded and cells were washed once with $3 \mathrm{ml}$ PBS on ice. Each cell pellet was resuspended in $100 \mu 1$ PBS, stained with $10 \mu 1$ propidium iodide (PI) and $4 \mu 1$ Annexin V-fluorescein isothiocyanate $(20 \mu \mathrm{g} / \mathrm{ml})$ and incubated on ice for $30 \mathrm{~min}$ in the dark. Cell apoptosis was analyzed by flow cytometry (Epics XL; Beckman Coulter, Inc., Brea, CA, USA), and data were analyzed using a FlowJo Software 7.6 (Tree Star, Inc., Ashland, OR, USA).

Western blot analysis. MSCs were harvested and lysed in RIPA buffer (Sigma-Aldrich; Merck KGaA; cat. no. 20-188) at $4^{\circ} \mathrm{C}$. Following centrifugation $\left(14,000 \mathrm{xg}\right.$ for $15 \mathrm{~min}$ at $\left.4^{\circ} \mathrm{C}\right)$, the protein concentration was determined using the Bradford method (Beyotime Institute of Biotechnology, Nantong, China) according to the manufacturer's protocol. A total of $20 \mu \mathrm{g}$ total protein sample was separated by $10 \%$ SDS-PAGE (Hangzhou Fude Biological Technology Co., Ltd., Hangzhou, China) and transferred onto a nitrocellulose membrane. The nitrocellulose membrane was blocked with 5\% nonfat milk for $1 \mathrm{~h}$ at room temperature then incubated with antibodies against $\mathrm{p} 65$ NF-кB (Abcam; cat. no. ab16502; $0.5 \mu \mathrm{g} / \mathrm{ml}$ ), PCNA (Abcam; cat. no. ab18197; $1 \mu \mathrm{g} / \mathrm{ml}$ ) and Fas (Abcam; cat. no. ab82419; $1: 1,000)$ at $4^{\circ} \mathrm{C}$ overnight. The nitrocellulose membrane was then incubated with anti-rabbit IgG (Abcam; cat. no. ab191866; 1:500) secondary antibodies, for $1 \mathrm{~h}$ at room temperature. Finally, protein was detected using a 3,3'-diaminobenzidine kit (Beijing Solarbio Science \& Technology Co., Ltd., Beijing, China). GAPDH served as the internal control.

ELISA. The expression levels of VEGF (R\&D Systems, Inc., Minneapolis, MN, USA; cat. no. RRV00), CD45 (G-Biosciences, St Louis, MO, USA; cat. no. 50-148-9078), IL-10 (Invitrogen; Thermo Fisher Scientific, Inc.; cat. no. ERIL10) and INF- $\gamma$ (R\&D Systems, Inc.; cat. no. RIF00) in the aqueous humor of rats were determined using an ELISA kit. Measurements were conducted according to the manufacturer's protocol. A microplate reader was used to determine the optical densities and data were presented as means of triplicate wells. 
Table I. Expression levels of miR146a in each group (means \pm standard deviation).

\begin{tabular}{lcccr}
\hline Group & Control, $\%$ & Normal MSCs, $\%$ & miR146a-low MSCs, \% & miR146a-high MSCs, \% \\
\hline miR146a & $100.0 \pm 11.2$ & $97.9 \pm 10.5$ & $67.5 \pm 9.1^{\mathrm{a}}$ & $137.5 \pm 15.9^{\mathrm{b}}$ \\
\hline
\end{tabular}

${ }^{\mathrm{a}} \mathrm{P}<0.001$ and ${ }^{\mathrm{b}} \mathrm{P}<0.01$ vs. Control. miRNA146a, microRNA 146a; MSC, mesenchymal stem cell.

Histological analysis. Histological analysis of corneal tissue samples was performed by hematoxylin and eosin (H\&E) staining. Rats were administered with a subconjunctival injection of MSCs ( $1 \times 10^{7}$ cells), or PBS for the control, through the tail vein. The rats were subsequently sacrificed, following 4 weeks of treatment, for histological examination. Corneal tissue samples were preserved in $4 \%$ formaldehyde solution at room temperature, dehydrated and embedded in paraffin. The $5-\mu \mathrm{m}$ paraffin sections were immersed in distilled water according to routine strategy. H\&E staining was conducted as follows: Washing with running water for $30 \mathrm{~min}$, dehydration in $90 \%$ alcohol for $5 \mathrm{~min}$ and eosin staining for $3 \mathrm{~min}$ at room temperature. Then pictures were captured under x10 magnification using a light microscope (Leica Upright Microscope; Leica Microsystems GmbH, Wetzlar, Germany).

Statistical analysis. Each experiment was repeated three times independently. Data are presented as means \pm standard deviation. Data analyses were conducted using GraphPad Prism 6.0 software (GraphPad Software, Inc., La Jolla, CA, USA). Statistical significance was determined by using an analysis of variance followed by least significant difference post hoc assessment $(\alpha=0.05)$ and $P<0.05$ was considered to indicate a statistically significant difference.

\section{Results}

miR146a expression levels in MSCs from each group. As shown in Table I, the results of qPCR indicate that there was no significant difference between the Normal MSCs group and the Control group, and the expression level of miR146a in the miR146a-low MSCs was significantly decreased to $67.5 \pm 9.1 \%$ $(\mathrm{P}<0.001$ vs. Normal MSCs), the expression level of miR146a in miR146a-high MSCs was increased to $137.5 \pm 15.9 \%(\mathrm{P}<0.01$ vs. Normal MSCs).

miR146a-high MSCs improve the cell viability of MSCs following alkali burn. An MTT assay was performed to confirm the cell viability of each group. As shown in Fig. 1, cell viability was significantly decreased following alkali burn, while the cell viability of the miR146a-low MSCs group was further decreased when compared with that of Normal MSCs. However, the cell viability of the miR146a-high MSCs group was restored, and significantly greater than that of the Normal MSCs group.

miR146a-high MSCs inhibited apoptosis of MSCs following alkali burn. As shown in Fig. 2, the percentage of apoptosis was detected using Annexin V/PI double staining in each group. The results indicate that cells are significantly apoptotic

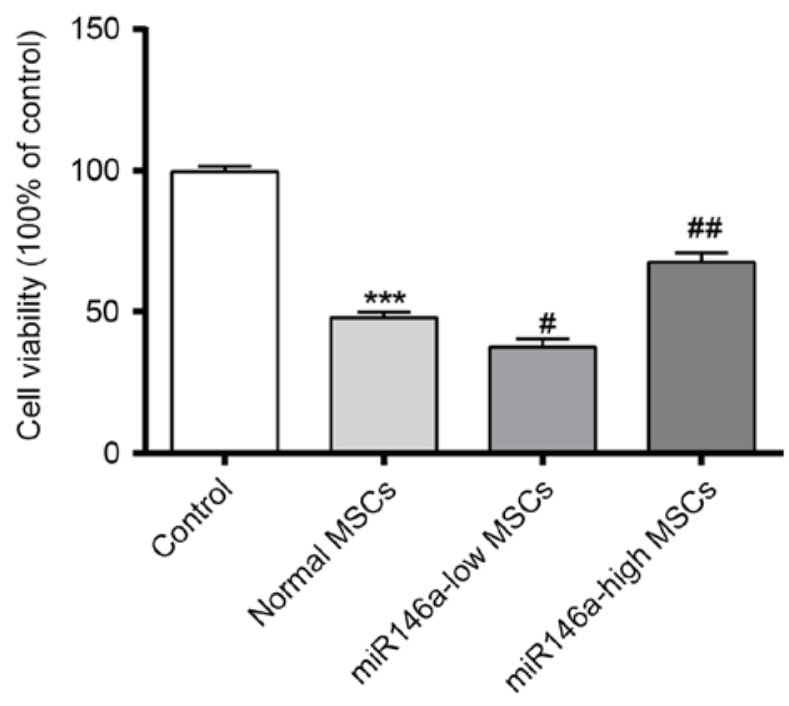

Figure 1. Expression levels of miR146a in each group on the effect of cell viability of MSCs. ${ }^{* * *} \mathrm{P}<0.001$ vs. Control; ${ }^{\#} \mathrm{P}<0.05$ vs. Normal MSCs; ${ }^{\# \#} \mathrm{P}<0.01$ vs. Normal MSCs. miR146a, microRNA 146a; MSC, mesenchymal stem cell.

following alkali burn; furthermore, the percentage of apoptotic cells in the miR146a-low MSCs group was significantly increased. However, the apoptosis ratio of the miR146a-high MSCs group was suppressed, and significantly reduced when compared with the Normal MSCs group.

Expression levels of $p 65 \mathrm{NF}-\kappa \mathrm{B}, \mathrm{PCNA}$ and Fas in MSCs following alkali burn. As shown in Fig. 3A and B, western blot results demonstrated that expression levels of p65 NF- $\kappa \mathrm{B}$ and PCNA were decreased in MSCs following alkali burn, although the expression level of Fas appears to be increased. p65 NF- $\kappa \mathrm{B}$ and PCNA were significantly decreased in the miR146a-low MSCs group, and Fas expression was increased to a certain degree, although the difference was not significant when compared with the Normal MSCs group. The levels of expression of p65NF- $\mathrm{B}$ and PCNA in the miR146a-high MSCs group were recovered to a certain degree, while the expression level of Fas was suppressed; the differences were significant when compared with the Normal MSCs group.

miR146a-high MSCs group demonstrated improved corneal opacity and enhanced the inhibition of neovascularization. The score of corneal opacity severity indicated that, after 1,2, 3 and 4 weeks of treatment, the degree of corneal opacity in the Normal MSCs group was significantly improved, although the cornea opacity in the miR146a-low MSCs group was suppressed, and the miR146a-high MSCs group demonstrated enhanced improvement of corneal opacity (Table II). The 
A

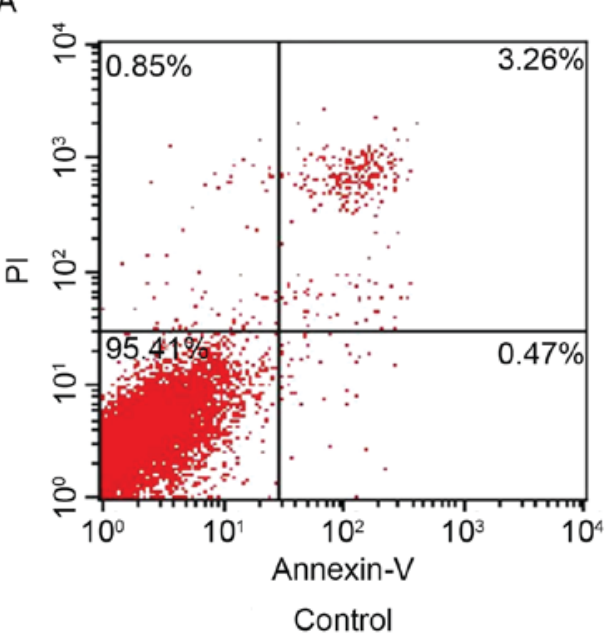

C

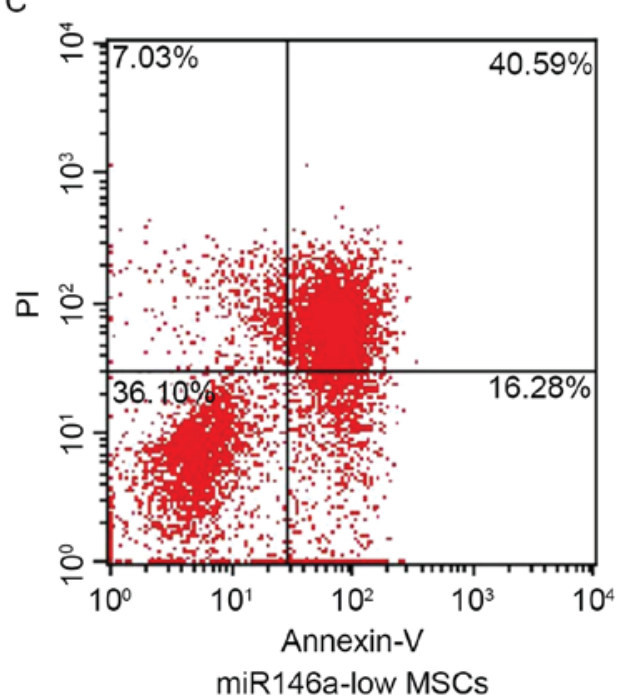

B

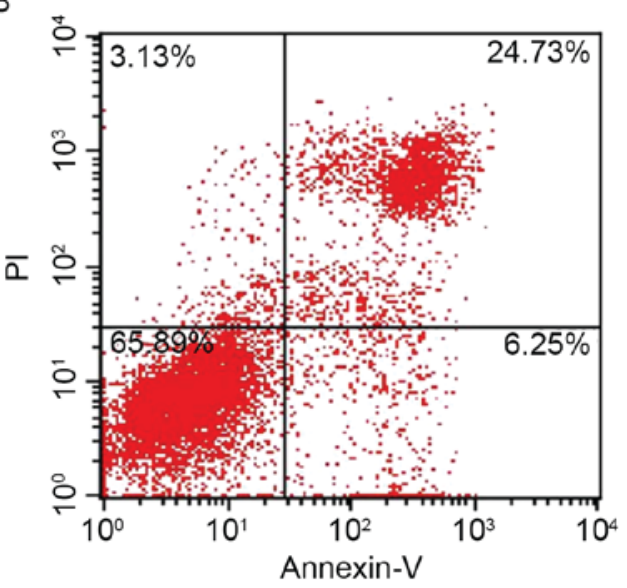

Normal MSCs

D

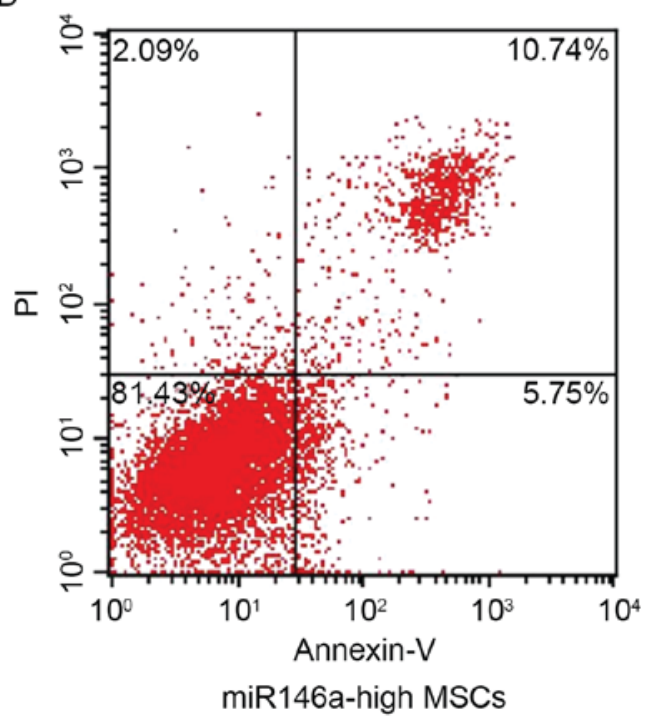

Figure 2. Expression levels of miR146a in each group and the effect on apoptosis. The apoptotic rate of normal MSCs (B), miR146a-low MSCs (C), miR146a-high MSCs (D) after miR146a-high MSCs after alkali burn, MSCs without any treatment as control (A). The upper right plus with lower right is the total apoptotic rate. miR146a, microRNA 146a; MSC, mesenchymal stem cell; PI, propidium iodide.

A P65NF-KB

Fas

PCNA

GAPDH

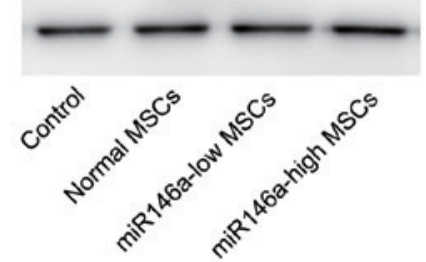

B

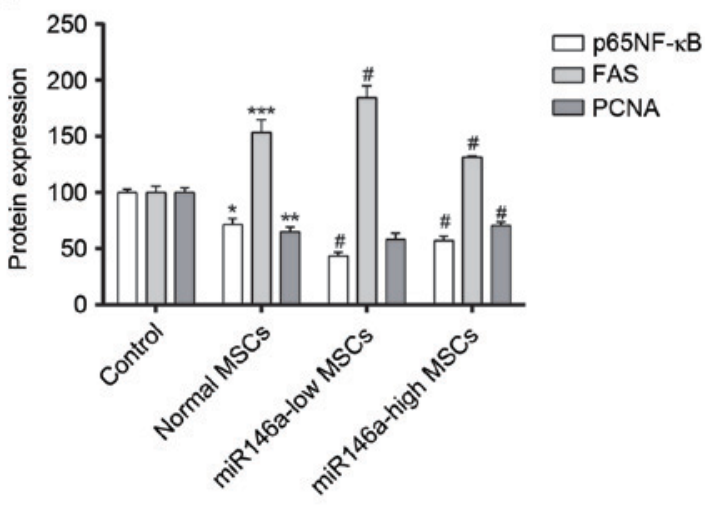

Figure 3. Expression levels of miR146a in each group and the effect on the expression levels of p65 NF-kB, PCNA and Fas in MSCs. (A) Western blotting was used to detect levels of expression of p65 NF- $\mathrm{kB}$, PCNA and Fas in MSCs. GAPDH served as the internal control. (B) Quantification of p65 NF- $\mathrm{kB}$, PCNA and Fas protein expression in MSCs. ${ }^{*} \mathrm{P}<0.05,{ }^{* *} \mathrm{P}<0.01$ and ${ }^{* * *} \mathrm{P}<0.001$ vs. Control; ${ }^{*} \mathrm{P}<0.05$ vs. Normal MSCs. miR146a, microRNA 146a; MSC, mesenchymal stem cell; NF- $\mathrm{kB}$, nuclear factor- $\mathrm{\kappa} B$; PCNA, proliferating cell nuclear antigen. 
Table II. Corneal opacity severity score of rats in each group.

Corneal opacity severity score

\begin{tabular}{lllll}
\cline { 2 - 4 } Group & 1 week & 2 weeks & 3 weeks & 4 weeks \\
\hline Control & $3.91 \pm 0.32$ & $3.88 \pm 0.31$ & $3.24 \pm 0.28$ & $2.92 \pm 0.24$ \\
Normal MSCs & $3.01 \pm 0.22^{\mathrm{a}}$ & $2.75 \pm 0.26^{\mathrm{a}}$ & $2.10 \pm 0.36^{\mathrm{a}}$ & $1.98 \pm 0.21^{\mathrm{a}}$ \\
miR146a-low MSCs & $3.38 \pm 0.37^{\mathrm{b}}$ & $3.16 \pm 0.25^{\mathrm{b}}$ & $2.69 \pm 0.27^{\mathrm{b}}$ & $2.33 \pm 0.34^{\mathrm{b}}$ \\
miR146a-high MSCs & $2.54 \pm 0.23^{\mathrm{b}}$ & $2.18 \pm 0.34^{\mathrm{b}}$ & $1.89 \pm 0.19^{\mathrm{b}}$ & $1.55 \pm 0.16^{\mathrm{b}}$ \\
\hline
\end{tabular}

${ }^{\mathrm{a}} \mathrm{P}<0.05$ vs. Control; ${ }^{\mathrm{b}} \mathrm{P}<0.05$ vs. Normal MSCs. MSC, mesenchymal stem cell; miRNA146a, microRNA $146 \mathrm{a}$.

Table III. Comparison of the neovascularization area of rats in each group.

\begin{tabular}{|c|c|c|c|c|}
\hline \multirow[b]{2}{*}{ Group } & \multicolumn{4}{|c|}{ Neovascularization area, $\mathrm{mm}^{2}$} \\
\hline & 1 week & 2 weeks & 3 weeks & 4 weeks \\
\hline Control & $10.2 \pm 1.22$ & $18.3 \pm 2.01$ & $16.7 \pm 1.78$ & $15.5 \pm 1.68$ \\
\hline Normal MSCs & $8.23 \pm 1.74^{\mathrm{a}}$ & $13.4 \pm 1.24^{\mathrm{b}}$ & $10.6 \pm 1.20^{\mathrm{b}}$ & $8.74 \pm 1.09^{b}$ \\
\hline miR146a-low MSCs & $9.26 \pm 1.18^{c}$ & $15.4 \pm 1.66^{\mathrm{c}}$ & $13.1 \pm 1.54^{\mathrm{c}}$ & $11.1 \pm 1.07^{\mathrm{c}}$ \\
\hline miR146a-high MSCs & $6.64 \pm 0.77^{\mathrm{d}}$ & $10.2 \pm 1.04^{\mathrm{c}}$ & $6.36 \pm 0.87^{\mathrm{d}}$ & $3.96 \pm 0.49^{\mathrm{d}}$ \\
\hline
\end{tabular}

${ }^{\mathrm{a}} \mathrm{P}<0.05$ and ${ }^{\mathrm{b}} \mathrm{P}<0.01$ vs. Control; ${ }^{\mathrm{C}}<0.05$ and ${ }^{\mathrm{d}} \mathrm{P}<0.01$ vs. Normal MSCs. MSC, mesenchymal stem cell; miRNA146a, microRNA 146a.

results of the neovascularization show that after weeks 1-4 of treatment, the neovascularization was normal. MSCs was significantly inhibited, but the inhibition of neovascularization was weakened in the miR146a-low MSCs group, and the miR146a-high MSCs group demonstrated enhanced inhibition of neovascularization (Table III).

Levels of VEGF secretion in the aqueous humour and inflammation-associated cytokine expression levels in rat corneal tissue samples following alkali burn. Fig. 4 demonstrates results of 4 weeks following the alkali burn surgery being performed. The content of VEGF was significantly decreased in the Normal MSCs group and the content of VEGF was significantly higher in the miR146a-low MSCs group compared with the Normal MSCs group, while the content of VEGF in the miR146a-high MSCs group was significantly lower than in the Normal MSCs group. In addition, evaluation of the corneal tissue inflammation factors, CD45 and IFN- $\gamma$ demonstrated a consistent trend, whereas the change in the concentration of IL-10 was the opposite (indicating the special status of IL-10, which requires further investigation). This indicated that miR146a may inhibit the expression levels of VEGF, CD45 and IFN- $\gamma$, while enhancing the expression level of IL-10. These results indicate that the miR146a-high MSCs group exerted a better effect on inhibiting the inflammation when compared with the Normal MSCs group.

Histological observation of the rat corneas following treatment. After four weeks of treatment, morphological observation of corneal tissue samples was conducted by H\&E staining (Fig. 5). In the control group, the corneal surface was covered with epithelial cells, no edema in the stroma, collagen fibers neatly arranged and a large number of visible novel blood vessels are evident in the corneal stroma. In the normal MSCs group the epithelial corneal wound was healed, there was no edema in the stroma and the collagen fibers were neatly arranged, however only a few of visible novel blood vessels are visible in the corneal stroma. In the miR146a-low MSCs group, the corneal surface was covered with epithelial cells with no edema in the stroma, the collagen fibers neatly arranged and a mass of visible novel blood vessels present in the corneal stroma. In the miR146a-high MSCs group, the epithelial corneal wound was healed, there was no edema in stroma, collagen fibers were tightly arranged and there were only a few visible novel blood vessels in the corneal stroma.

\section{Discussion}

Corneal alkali burn is a common clinical ophthalmology disease, the treatment of which is quite difficult and the prognosis is poor. Previous studies of the underlying mechanism of the injury have been performed $(19,20)$. MSCs are an important cellular component of the hematopoietic microenvironment, which proliferate and differentiate into a variety of tissues, and have low immunogenicity. Furthermore, MSCs have a high degree of proliferation, self-renewal and pluripotency (21). Clinical trials confirmed that MSCs may be used in tissue repair (22). For the osteogenic differentiation of MSCs, 

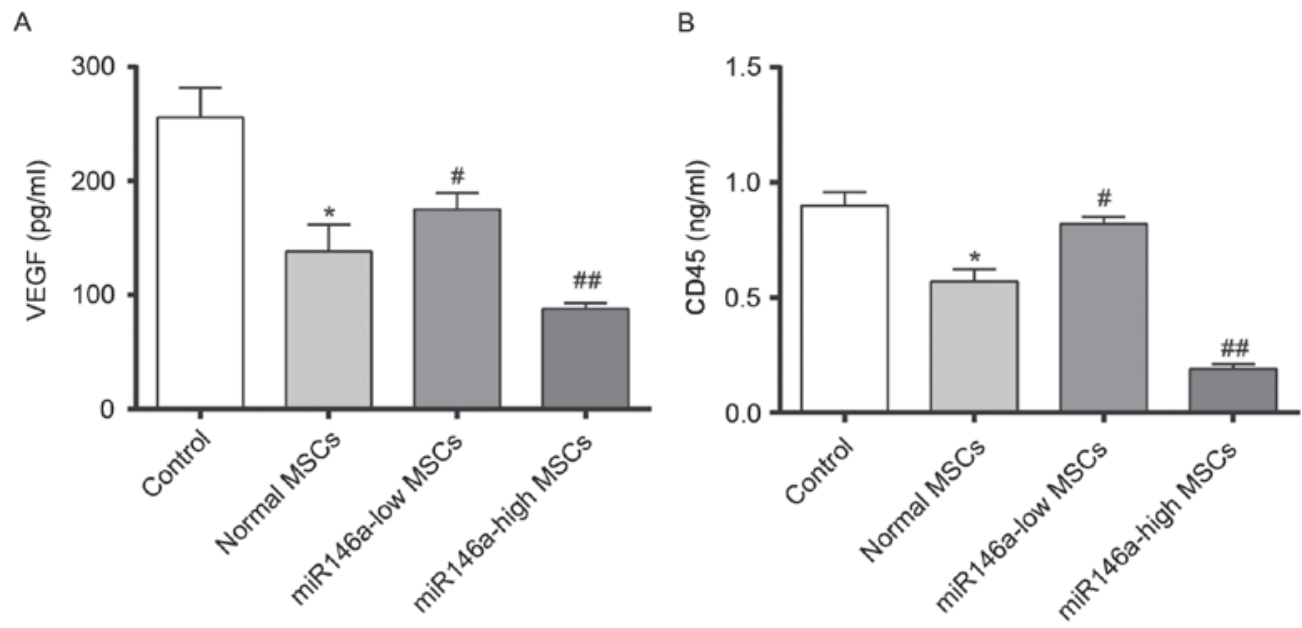

C

D
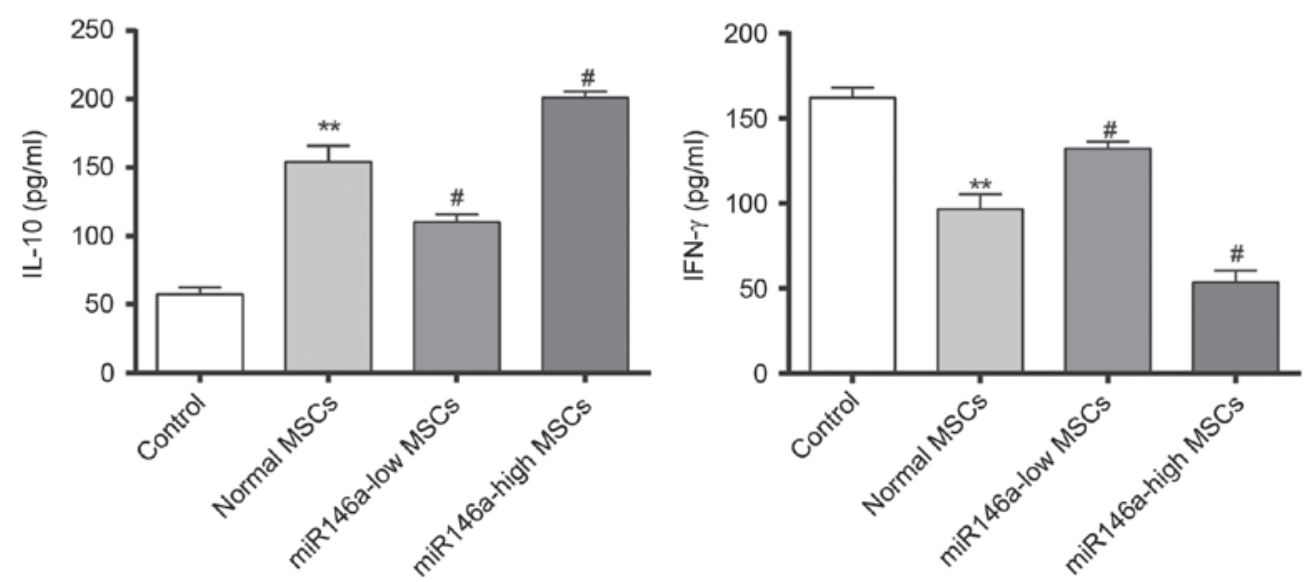

Figure 4. Change of VEGF in the aqueous humor and inflammation factor levels in the corneal tissue samples from rats in each group. Quantification of (A) VEGF, (B) CD45, (C) IL-10 and (D) IFN- $\gamma$ secretion in MSCs. ${ }^{*} \mathrm{P}<0.05$ and ${ }^{* *} \mathrm{P}<0.01$ vs. Control; ${ }^{*} \mathrm{P}<0.05$ and ${ }^{\# \#} \mathrm{P}<0.01$ vs. Normal MSCs. VEGF, vascular endothelial growth factor; IFN- $\gamma$, interferon- $\gamma$; IL, interleukin; MSC, mesenchymal stem cell.

A

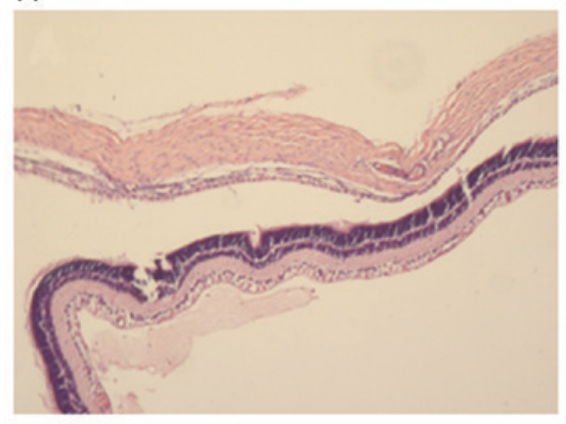

C

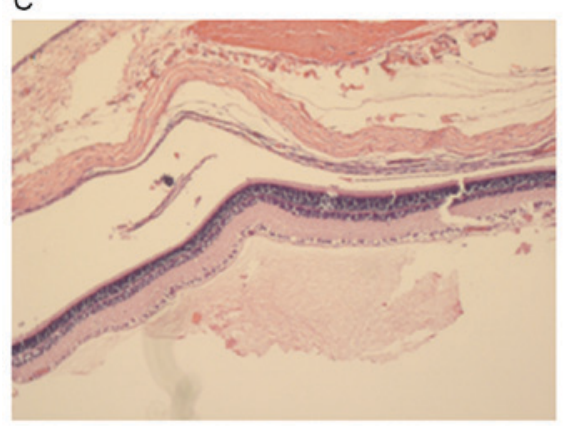

B

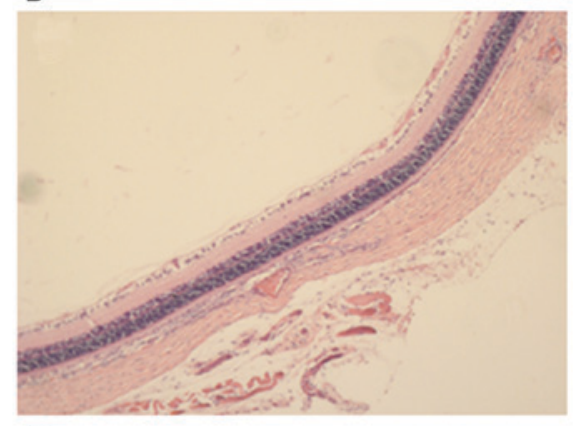

D

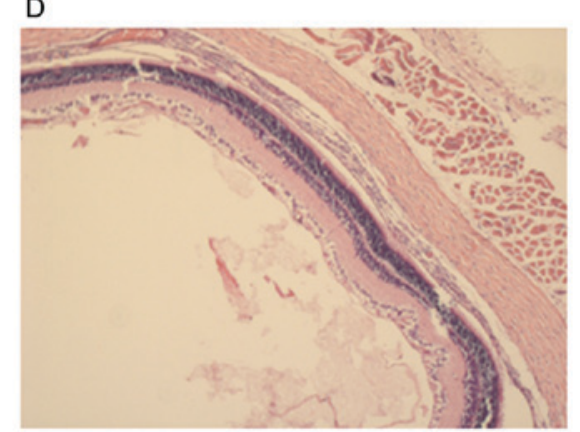

Figure 5. Hematoxylin and eosin staining following treatment of corneal alkali burn in each group. The H\&E staining of rat corneas of (A) control, (B) normal MSCs, (C) miR146a-low MSCs and (D) miR146a-high MSCs groups following 4 weeks treatment. miRNA146a, microRNA 146a. 
multiple cytokines and signaling pathways are involved in the regulation of differentiation.

In the current study, MSCs were transfected with lentiviral recombined miR146a genes. In the current assay, SD rats were used to establish corneal alkali burn models to evaluate the effects of miR146a-high MSCs. The data demonstrated that miR146a-high MSCs inhibited cell apoptosis in corneal alkali burn rats. In addition, miR146a-high MSCs inhibited the expression of p $65 \mathrm{NF}-\kappa \mathrm{B}$ and PCNA, and promoted the expression of Fas in corneal alkali burn rats. The result implied that miR146a-high MSCs produced a strong repair effect and provided protection (23). These results demonstrated that genetically modified miR146a-high MSCs represent a promising strategy for corneal alkali burn therapeutic strategies.

One or four weeks after transplantation, corneal haze and the neovascular situation were observed under a slit lamp (the growth time, length and number of CNV were recorded, and the length and area of CNV were calculated as described previously (24). The corneal alkali burn scoring (cornea scoring criteria following alkali burn) was also evaluated according to the standard reference (25). Strong inhibition of CNV in rats treated with miR146a-high MSCs was observed. Thus, it was inferred that miR146a-high MSCs result in high repair effects, which may be due to the miR146a. Thus, further studies are required to determine the role of miR146a. As shown in Fig. 4, decreased expression levels of VEGF, CD45 and IFN- $\gamma$ were observed in the miR146a-high MSCs group rats. CD45 and IFN- $\gamma$ have been demonstrated to be inflammation-associated cytokines $(26,27)$. Therefore, miR146a promotes repair of tissues via inhibited secretion of CD45 and IFN- $\gamma$. Whereas the expression level of IL-10 was the opposite, which was a notable finding.

In conclusion, the present study indicated that miR146a in MSCs induced a powerful protective and repair effect. Notably, miR146a directly decreased the expression level of p65 NF- $\kappa$ B and PCNA, and inhibited apoptosis, inflammatory cytokine secretion and $\mathrm{CNV}$ in corneal alkali burn rats. The level of corneal opacity also improved significantly in rats treated with miR146a-high MSCs. These results imply that MSCs genetically modified with miR146a may serve as an effective therapeutic strategy for corneal alkali burn. The present study still has limitations, for example, the detailed signaling pathway requires further investigation, and large animal experiments also are required to determine the role of miR146a-high MSCs in treating corneal alkali burns.

\section{Acknowledgements}

Not applicable.

\section{Funding}

The present study was supported by Zhejiang Provincial Natural Science Foundation of China (grant nos. LY16H120004, LY16H110002 and LY14H020005).

\section{Availability of data and materials}

The analyzed data sets generated during the study are available from the corresponding author on reasonable request.

\section{Authors' contributions}

ZJ designed this study. XL and JL performed all the experiments. LY helped to collect data. JP helped to organize figures. YZ helped with analysis and interpretation of data.

\section{Ethics approval and consent to participate}

The present study was approved by the veterinary ethics committee of Zhejiang (Wenzhou, China).

\section{Consent for publication}

Not applicable.

\section{Competing interests}

The authors declare they have no competing interests.

\section{References}

1. Welling JD, Pike EC and Mauger TF: Alkali burn of the ocular surface associated with a commonly used antifog agent for eyewear: Two cases and a review of previous reports. Cornea 35: 289-291, 2016.

2. Saud EE, Moraes HV Jr, Marculino LG, Gomes JA, Allodi S and Miguel NC: Clinical and histopathological outcomes of subconjunctival triamcinolone injection for the treatment of acute ocular alkali burn in rabbits. Cornea 31: 181-187, 2012.

3. Hua MT and Betz P: Descemet membrane detachment after alkali ocular surface burn. Bull Soc Belge Ophtalmol: 85-86, 2010.

4. Nishiwaki-Dantas MC, Dantas PE and Reggi JR: Ipsilateral limbal translocation for treatment of partial limbal deficiency secondary to ocular alkali burn. Br J Ophthalmol 85: 1031-1033, 2001.

5. Ke Y, Wu Y, Cui X, Liu X, Yu M, Yang C and Li X: Polysaccharide hydrogel combined with mesenchymal stem cells promotes the healing of corneal alkali burn in rats. Plos One 10: e0119725, 2015.

6. Cox CD, Nakayama Y, Nomura $T$ and Martinac B: The evolutionary 'tinkering' of MscS-like channels: Generation of structural and functional diversity. Pflugers Arch 467: 3-13, 2015.

7. Dumitru CA, Hemeda H, Jakob M, Lang S and Brandau S: Stimulation of mesenchymal stromal cells (MSCs) via TLR3 reveals a novel mechanism of autocrine priming. Faseb J 28: 3856-3866, 2014.

8. Lemos DR, Eisner C, Hopkins CI and Rossi FMV: Skeletal muscle-resident MSCs and bone formation. Bone 80: 19-23, 2015.

9. Rowe I, Anishkin A, Kamaraju K, Yoshimura K and Sukharev S: The cytoplasmic cage domain of the mechanosensitive channel $\mathrm{MscS}$ is a sensor of macromolecular crowding. J Gen Physiol 143: 543-557, 2014.

10. Bhoj M, Zhang C and Green DW: A first step in de novo synthesis of a living pulp tissue replacement using dental pulp MSCs and tissue growth factors, encapsulated within a bioinspired alginate hydrogel. J Endod 41: 1100-1107, 2015.

11. Langroudi L, Hassan ZM, Soleimani M and Hashemi SM: Tumor associated mesenchymal stromal cells show higher immunosuppressive and angiogenic properties compared to adipose derived MSCs. Iran J Immunol 12: 226-239, 2015.

12. Bajpai I, Kim DY, Kyong-Jin J, Song IH and Kim S: Response of human bone marrow-derived MSCs on triphasic Ca-P substrate with various HA/TCP ratio. J Biomed Mater Res B Appl Biomater 105: 72-80, 2017.

13. Naqvi AR, Zhong S, Dang H, Fordham JB, Nares S and Khan A: Expression profiling of LPS responsive miRNA in primary human macrophages. J Microb Biochem Technol 8: 136-143, 2016.

14. Li D, Mou W, Luo Z, Li L, Limwachiranon J, Mao L and Ying T: Developmental and stress regulation on expression of a novel miRNA, Fan-miR73, and its target ABI5 in strawberry. Sci Rep 6: 28385, 2016. 
15. Wang P, Xu J, Hou Z, Wang F, Song Y, Wang J, Zhu H and Jin H: miRNA-34a promotes proliferation of human pulmonary artery smooth muscle cells by targeting PDGFRA. Cell Prolif 49: 484-493, 2016.

16. Zibara K, Awada Z, Dib L, El-Saghir J, Al-Ghadban S, Ibrik A, El-Zein N and El-Sabban M: Anti-angiogenesis therapy and gap junction inhibition reduce MDA-MB-231 breast cancer cell invasion and metastasis in vitro and in vivo. Sci Rep 5: 12598, 2015.

17. Livak KJ and Schmittgen TD: Analysis of relative gene expression data using real-time quantitative PCR and the 2(-Delta Delta C(T)) method. Methods 25: 402-408, 2001.

18. Ji YB and Yu L: In vitro analysis of the role of the mitochondrial apoptosis pathway in CSBE therapy against human gastric cancer. Exp Ther Med 10: 2403-2409, 2015.

19. Giacomini C, Ferrari G, Bignami F and Rama P: Alkali burn versus suture-induced corneal neovascularization in C57BL/6 mice: An overview of two common animal models of corneal neovascularization. Exp Eye Res 121: 1-4, 2014.

20. Anderson $C$, Zhou $Q$ and Wang S: An alkali-burn injury model of corneal neovascularization in the mouse. J Vis Exp 86: e51159, 2014.

21. Nikiforou M, Willburger C, De Jong AE, Kloosterboer N, Jellema RK, Ophelders DR, Steinbusch HW, Kramer BW and Wolfs TG: Global hypoxia-ischemia induced inflammation and structural changes in the preterm ovine gut which were not ameliorated by mesenchymal stem cell treatment. Mol Med 22, 2016.
22. Liang X, Zhang L, Wang S, Han Q and Zhao RC: Exosomes secreted by mesenchymal stem cells promote endothelial cell angiogenesis by transferring miR-125a. J Cell Sci 129: 2182-2189, 2016.

23. Xu X, Yan C, Kossmann BR and Ivanov I: Secondary interaction interfaces with PCNA control conformational switching of DNA polymerase PolB from polymerization to editing. J Phys Chem B 120: 8379-8388, 2016.

24. Su P, Wang Y, Cooper DN, Zhu W, Huang D, Férec C, Wang Y and Chen JM: Disclosing the hidden structure and underlying mutational mechanism of a novel type of duplication CNV responsible for hereditary multiple osteochondromas. Hum Mutat 36: 758-763, 2015.

25. Moro C, Cornette R, Vieaud A, Bruneau N, Gourichon D, Bed'hom B and Tixier-Boichard M: Quantitative effect of a CNV on a morphological trait in chickens. Plos One 10: e0118706, 2015.

26. Ikeda H, Old LJ and Schreiber RD: The roles of IFN gamma in protection against tumor development and cancer immunoediting. Cytokine Growth Factor Rev 13: 95-109, 2002.

27. Baer C, Squadrito ML, Laoui D, Thompson D, Hansen SK, Kiialainen A, Hoves S, Ries CH, Ooi CH and De Palma M: Suppression of microRNA activity amplifies IFN-gamma-induced macrophage activation and promotes anti-tumour immunity. Nat Cell Biol 18: 790-802, 2016.

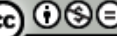

This work is licensed under a Creative Commons Attribution-NonCommercial-NoDerivatives 4.0 International (CC BY-NC-ND 4.0) License. 